\title{
The Recognition of Polynomial Position and Orientation through the Finite Polynomial Discrete Radon Transform
}

\author{
Ines Elouedi, Régis Fournier, Amine Naït-Ali, and Atef Hamouda \\ Faculté des sciences de Tunis, (LIPAH) Tunisia \\ Université Paris-Est Créteil, (LISSI) France
}

\begin{abstract}
In this paper, we propose to accurately detect from an image curvilinear features that can be approximated by polynomial curves. Having the a priori knowledge of a polynomial parameters (coefficients and degree), we give the possibility to recognize both the orientation and the position of the polynomial (if it exists) in the given image. For this objective, we present a new approach titled "The Finite Polynomial Discrete Radon Transform" (FPDRT) that maps the initial image into a Radon space where each point presents the amount of evidence of the existence of a polynomial at the same position. The FPDRT sums the pixels centered on a polynomial and stores the result at the corresponding position in the Radon space. The FPDRT extends the formalism of the Finite Discrete Radon Transform(FRT) which is restricted to project the image along straight lines of equation $y=m x+t$ where $m$ and $t$ are integers. Our method generalizes FRT by projecting the image with respect to polynomials of equation $y=m x^{n}+t$ where $m, n$ and $t$ are integers. The FPDRT method is exactly invertible, requires only arithmetic operations and is applicable to $p \times p$ sized images where $p$ is a prime number. Several applications are allowable by the FPDRT such as fingerprint, palm print biometric applications and multi directional roads recognition.
\end{abstract}

Keywords: polynomial curves,Finite Polynomial Discrete Radon Transform, Radon space.

\section{Introduction}

One of the most basic processes in image analysis is the extraction of primitives, such as lines and curves from an image. In this paper we focus on curves detection, or more precisely, on detection of curves that can be modelized by polynomials. One method that has proved successful for pattern recognition is the Radon transform (RT). This transform projects a two dimensional image along straight lines with respect to different directions as it is expressed in the following equation:

$$
R(\rho, \theta)=\int_{-\infty}^{+\infty} \int_{-\infty}^{+\infty} \delta(\rho-x \cos \theta-y \sin \theta) d x d y .
$$

A. Petrosino (Ed.): ICIAP 2013, Part II, LNCS 8157, pp. 249-258, 2013.

(C) Springer-Verlag Berlin Heidelberg 2013 
$\delta(n)$ is the kronecker delta function. The parameter $\rho$ is the shortest distance between the origin of the coordinate system and the line, and $\theta$ is the angular orientation parameter of the line. The given result of the transform is the parameters space $R(\rho, \theta)$ (denoted also by the Radon space) where a high valued pixel(peak) presents a high probability of the existence of a straight line mapped by the same parameters as the peak coordinates. Therefore, straight lines detection is then reduced to the research of peaks in the parameters space. This work generalizes the classical Radon transform by projecting images along polynomials. Then, a peak in the resulting Radon space reveals the existence of a polynomial and denotes its position and orientation in the original image.In this paper, we present the Finite Polynomial Discrete Radon Transform (FPDRT) which is a discrete approach that extends the classical Radon transform to recognize polynomial curves from the Radon space. Furthermore, the FPDRT allows an exact inversion of the Radon space in such a way that the initial image can be exactly reconstructed from the Radon space. This paper is organized as follows. In Section 2, we highlight some related works. In Section 3, we define the FPDRT followed by its inversion algorithm presented in section4. Experiments are then presented in section 5 to illustrate the performance of the FPDRT. In final, a conclusion of our paper is provided in section 6 .

\section{Related Works}

The use of the Radon Transform (RT) in pattern recognition is not recent. In fact, the Radon Transform is well known in the literature as an efficient method for segments detection as it has the capacity to transform thin lines to high valued peaks in the Radon space [1. This propriety gives rise to many applications related to the computer vision such as the detection of ship wake from satellite images [2], 3], the centerline detection, line width estimation [4] and the rectangular buildings contours extraction [5]. Although the RT was mainly investigated for segments detection, its use was extended to recognize more sophisticated features, namely: rectangles, spots of circular and square shapes. We give as example the work of Magli et. Al [ 6 where the authors apply the RT and then the continuous wavelet transform to analyze the wave shapes of peaks exceeding predetermined threshold. The wave shapes differ according to the nature of the pattern itself. Another work using the RT for more general pattern recognition is the study of Hmida et. Al [7] where the authors extract rectangular buildings from high resolution satellite images. For this purpose, the authors performed a one dimensional curve along the $\rho$ direction for each angle $\theta$ (cf. Eq 1), denoted by $R_{\theta}$ curve. Then, to enhance the rectangular shape, a comparison between each curve $R_{\theta}$ and a perfect curve corresponding to an ideal rectangle is performed. The retained curve is the one that shows the highest matching level. In addition to the extraction of buildings position and orientation, this method enables the estimation of the rectangles width. As we have shown, the RT by itself is not able to directly recognize generalized patterns as is the case of segments features. It requires further complicated processing to make the shapes 
recognition task possible. Therefore, several researchers proposed to generalize the Radon transform by projecting the image along arbitrary curves and shapes. This new transform sums the pixels centered into the curves and concentrates the result at the corresponding parameters in the Radon space. Hence, curves give rise to high valued peaks in the radon space; the coordinates of the local maximum are the parameters of the curve along which the projection was performed.Deans was the first to introduce the generalized Radon transform [8]. Peter Toft in his thesis [9] defined the generalized Radon Transform and proposed a discrete fast algorithm to estimate curves parameters and tested his algorithm in synthetic hyperbolas curves. Hendriks et.Al in [10] proposed a discrete generalized Radon transform for the detection of hollow hyper-spheres in D-dimensional space. However, the proposed generalized Radon transforms in the above works [8, 9] and [10] are not reversible. This is considered as a disadvantage since the geometric transforms are in general reversible in a such way that the initial image can be recovered from the Radon space and vice versa. Beylkin [11] presented a discrete version of the classical Radon transform and showed that the transform is exactly invertible if some conditions are verified. He showed that his transform holds also for generalized curves but his approach does not yield the multi orientation property as the classical RT. This inconvenience makes the transform inefficient for pattern recognition field where the features may be distributed with correspondence to any possible orientation. In this paper, we present a new approach aiming to generalize the classical Radon Transform by projecting the image within polynomial curves. This transform extends the exactly invertible classical Finite Radon Transform [12, is a multi oriented transform and also exactly invertible.

\section{The Finite Polynomial Discrete Radon Transform}

The FPDRT generalizes the FRT method defined by Flusser et. Al in [12] in order to hold polynomial curves. The FRT is a discrete Radon Transform applied on images sized of $p \times p$ where $p$ is prime number. Starting from the assumption that the image is periodic within $p$ and thanks to some arithmetic operations, the method is exactly invertible. The transform is performed by projecting a digital image along straight lines of equation $y=m x+t$ where $m$ and $t$ are integers in $\mathbb{Z}_{p}$. The FPDRT extends the FRT to project the image with respect to polynomials of equation $y=m x^{n}+t$ where $m$ and $t$ are integers in $\mathbb{Z}_{p}$. Similarly to the FRT, our method is applied on digital images sized of $p \times p$ where $p$ is prime number. This restriction on the size of the image allows some arithmetic proprieties that lead to an exact invertible algorithm. However, if the FPDRT is needed for an image with an arbitrary size $M \times N$, the image may be padded out by zeros to achieve the nearest size $p \times p$ with $p$ is the smallest prime number that verifies $p \geq \sup \{M, N\}$.It is to note that the FPDRT transform also applies with a more generalized equation of polynomial $Q$ such that

$Q(x)=m x^{n}+a_{n-1} x^{n-1}+\ldots .+t$ where $a_{n-1}, a_{n-2}, \ldots, a_{1}$ are the remaining non- zero coefficient terms of $Q$. 
In the following two subsections, we describe the forward finite polynomial transform and the inverse algorithm for it.

\subsection{The Forward Finite Polynomial Discrete Radon Transform}

The forward FPDRT is the first stage in the approach. It generates the radon space presenting the polynomial projection result. The required $p \times p$ data is defined as the finite group under addition $\mathbb{Z}_{p}^{2}$ so that the $x$ and $y$ axes range both from 0 to $p-1$ and the image is periodic within $p$ in both the $x$ and $y$ directions as follows

$I(x+p, y+p)=I(x, y)$.

A projection $R_{m}(v, t)$ sums the pixels belonging to a polynomial mapped according to a leader term coefficient $m$ and intercepts $v$ and $t$ which denote respectively the $x$-axis and the $y$-axis translates. The equation of the forward projection is defined as follows:

$$
\begin{gathered}
R_{m}(v, t)=\sum_{x=0}^{p-1} \sum_{y=0}^{p-1} I(x, y) \times \delta\left(y-<m\left(<x-v>_{p}\right)^{n}+t>_{p}\right) . \\
=\sum_{x=0}^{p-1} I\left(x,<m\left(<x-v>_{p}\right)^{n}+t>_{p}\right) .
\end{gathered}
$$

$$
\text { for } m, t, v \in \mathbb{Z}_{p} \text {. }
$$

The notation $\langle x\rangle_{N}$ means $x(\bmod N)$. In addition to the horizontal projections $R_{m}$, we give the vertical projection $R_{0}^{\perp}$ defined as the sum made over the perpendicular polynomial curve of equation $x=\left\langle 0 y^{n}+v\right\rangle_{p}$. i.e.

$$
R_{0}^{\perp}(v)=\sum_{y=0}^{p-1} I(v, y) .
$$

Three properties emerge from the FPDRT projection:

a) Each projection $R_{m}$ sums exactly $p$ distinct points from the image I .

b) The pattern of sampled pixels by $R_{m}$ is unique for each $m$ in $\mathbb{Z}_{p}$. For a given fixed $v^{\prime}$ and $t^{\prime}$ in $\mathbb{Z}_{p}$ and for $m$ varying in $\mathbb{Z}_{p}$, all the projections $R_{m}\left(v^{\prime}, t^{\prime}\right)$ intersect only once at coordinates $\left(v^{\prime}, t^{\prime}\right)$.

c) For given fixed values $m^{\prime}$ and $v^{\prime}$ in $\mathbb{Z}_{p}$, all the projection functions $R_{m^{\prime}}\left(v^{\prime}, t\right)$, for $t$ in $\mathbb{Z}_{p}$ select distinct points from I .In other terms, The horizontally shifted polynomials are parallel and there is no intersection between them.

Considering the properties a) and c), it can be seen that, for fixed values $m$ and $v$ and for $t$ ranging in $\mathbb{Z}_{p}$, all pixels from $I(x, y)$ will be chosen just once by the projection functions $R_{m}(v, t)$. Therefore, 


$$
\sum_{t=0}^{p-1} R_{m}(v, t)=\sum_{x=0}^{p-1} \sum_{y=0}^{p-1}=\text { Isum. }
$$

where Isum is the total sum of the image pixels. The Fig. 1 presents an illustration of the FPDRT projections applied on a simple $5 \times 5$ sized image I: The curve projected here is a parabola of equation $y=m(x-v)^{2}+t(\bmod 5)$. The vertical axis is chosen to be the $\mathrm{x}$-axis and the $\mathrm{y}$-axis is the horizontal one. In this example, we have chosen the $\mathrm{x}$ values to range from $-(p-1) / 2$ to $(p-1) / 2$ instead of $[0, p-1]$. This change is made for pattern recognition considerations in order to extract patterns of pixels that fit the feature of parabolas which are symmetric curves. This change does not affect any of properties of the FPDRT transform. The vertical translate $v$ follows the values of $x$ and then ranges from $-(p-1) / 2$ to $(p-1) / 2$. The periodicity of the image is also preserved in a way that if $(x-v)$ exceeds $(p-1) / 2($ respectively $-(p-1) / 2)$, the vertical translate $v$ will be wrapped back to the beginning of the column i.e. at $-(p-1) / 2$ value ( respectively to the end of the column i.e. to $(p-1) / 2$ value ) in that way:

$$
\begin{aligned}
& \text { if }(x-v) \in]-p,-(p-1) / 2[\text {, then }(x-v)=(x-v)(\bmod p) \\
& \text { if }(x-v) \in](p-1) / 2, p[\text {, then }(x-v)=(x-v)(\bmod (-p)) .
\end{aligned}
$$

The horizontal translate $t$ follows the $y$ values and ranges from 0 to $p-1$. The shaded pixels from Fig.1.a present the set of summed pixels by $R_{1}(0,0)$ which corresponds to the parabola of equation $y=\left(x^{2}\right)(\bmod 5)$. When we horizontally shift the curve by a one pixel (i.e. when $t=1$ and the parabola equation is $\left.y=\left(x^{2}+1\right)(\bmod 5)\right)$, the projection function $R_{1}(0,1)$ sums the shaded pixels of the Fig.1.b. The pixels of coordinates $(-2,5)$ and $(2,5)$ are wrapped back by the $\bmod (5)$ respectively to $(-2,0)$ and $(2,0)$. It can be seen that each function $R_{1}(0,0)$ and $R_{1}(0,1)$ sums exactly 5 distinct pixels and since the corresponding two parabolas are parallel, the related sets of pixels do not intersect. When we translate the same curve vertically (i.e. for $v=1$ ), the periodicity of the image within the $x$-axis allows the pixel $(-2,4)$ to be selected (Fig.1.c). Fig.1.d shows the pattern of pixels selected by the vertical projection $R_{0}^{\perp}(1)$.

\subsection{The Inverse Polynomial Discrete Radon Transform}

The reconstruction of a point $I^{\prime}(v, t)$ consists in summing all the projections $R_{m}(v, t)$ as well as the vertical projection $R_{0}^{\perp}(v)$ that have already selected and summed the point $I(v, t)$. In fact, the property (b) states that for fixed values $t$ and $v$, all the projections $R_{m}(v, t)$ as well as the vertical projection $R_{0}^{\perp}(v)$ sample distinct patterns pixels and intersect only at the point $(v, t)$. Then, all the pixels of the initial image are summed just once except the point $(v, t)$ which is selected $(p+1)$ times ( $p$ times by the $R_{m}(v, t)$ projections and once by the vertical projection $R_{0}^{\perp}(v)$ ). Then, we can conclude that the total sum of the image pixels plus $p$ times the value of $I(v, t)$ is obtained by summing the projections $R_{m}(v, t)$ and $R_{0}^{\perp}(v)$. As the proprety c) states that the sum of the 

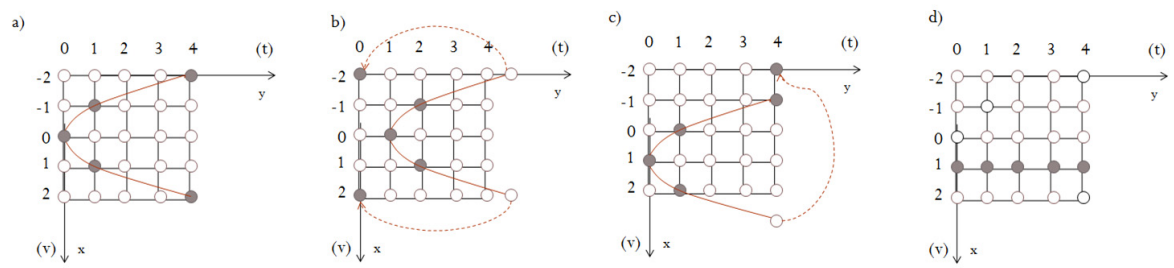

Fig. 1. Discrete parabolas in $5 \times 5$ arrays. a) shaded points lie on the center of a parabola of equation $y=x^{2}(\bmod 5)$. The selected pixels are summed to give $R_{1}(0,0)$. b) Shaded pixels correspond to the horizontally shifted parabola of equation $y=\left(x^{2}+\right.$ 1) $(\bmod 5)$.c) Shaded pixels lie on the vertically translated parabola of equation $y=$ $\left((x-1)^{2}\right)(\bmod 5)$. d) The vertical projection $R_{0}^{\perp}(1)$.

total pixels of the image can be found by summing all the parallel polynomials for a fixed $m$ and $v$, then we define the following equation as the reconstruction equation:

$$
I(v, t)=1 / p \times\left(\sum_{m=0}^{p-1} R_{m}(v, t)+R_{0}^{\perp}(v)-\text { Isum }\right) .
$$

Where $I$ sum $=\sum_{t=0}^{p-1} R_{m}(v, t)$, with $v, t \in \mathbb{Z}_{p}$.

\section{The Pattern Recognition Task}

In the classical Radon transform, the direction of straight lines along which the projection is processed is set by the angle $\theta$ (cf. Eq. 1). In the FRT method 12, the integer $m$ presents the gradient of the straight line $m=t g^{-1}(\theta)$. The variation of the grdient $m$ leads to the variation of the straight lines directions which preserves the multi orientation property of the classical Radon transform. In our case, the integer $m$ presents the leader term coefficient of the polynomial and thus the variation of $m$ leads to the variation of the scale of the polynomial and has no effect on its orientation. Nevertheless, the multi orientation property is required to our transform, in order to project the image according to different orientations which allows to answer to the pattern recognition requirements since a polynomial must be accurately extracted whatever its orientation in the image. Therefore, we have added the angle $\theta$ as a new parameter which sets the orientation of the polynomial projection.We express the multi-oriented FPDRT by the following equation where we apply the FPDRT on the $-\theta$-rotated image $I_{-\theta}$ instead of $I$ : 


$$
R_{m, \theta}(v, t)=\sum_{x=0}^{p-1} \sum_{y=0}^{p-1} I_{-\theta}(x, y) \times \delta\left(y-<m\left(<x-v>_{p}\right)^{n}+t>_{p}\right) .
$$

The inversion algorithm leads to the exact reconstruction of the rotated image $I_{-\theta}$. The initial image I can be found by the inverse rotation of $I_{-\theta}$.

\section{Experiments}

We have applied the FPDRT to synthetic and real images. Fig.2 shows the result of the FPDRT on a synthetic image containing two parabolas of equation $y=x^{2}$. The first whose vertex is positioned at $(0,0)$ was rotated according to the angle $\theta=45^{\circ}$ and the second was vertically shifted to the position $(10,0)$.we have varied the projection angle $\theta$ by increasing it by $5^{\circ}$ unit step in the range $\left[0^{\circ}, 180^{\circ}\right]$. The Radon space $R_{1,0^{\circ}}$ presented in Fig.2.b shows a peak at the parabola vertex position $(10,0)$. A peak at $(0,0)$ is also depicted in the Radon space $R_{1,45^{\circ}}$ (cf Fig.2.c ) corresponding to the vertex position of the rotated parabola.
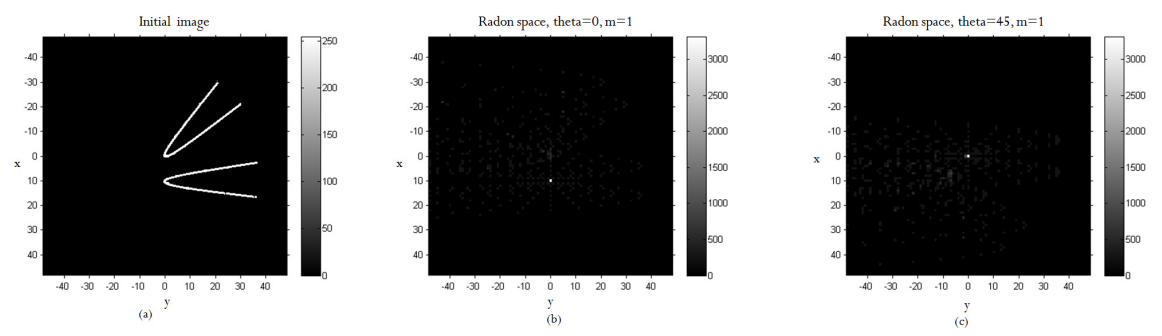

Fig. 2. a) : The initial image presenting two parabolas of equation $y=x^{2}$.the first parabola is translated by the vector $(\mathrm{v}, \mathrm{t})=(10,0)$ and the second is rotated with respect to the angle $\theta=45$.(b): The radon space for $\theta=0$ shows a peak at $(10,0)$.(c): The radon space for $\theta=45^{\circ}$ shows a peak at the position $(0,0)$ of the rotated parabola.

Fig. 3 presents the result of the FPDRT on a real fingerprint image. We have first binarized and thinned the initial image (Fig.3.b) on which we have applied the FPDRT by projecting it over parabolas of equation: $a y=m x^{2}$. Fig.3.c presents the radon space for $\theta=0^{\circ}, a=50$ and $m=3$. Clearly, a peak is depicted at the position of the vertex of the pointed parabola.

We have also tested the FPDRT on a real image presenting built arches (cf Fig.4.a). Fig.4.b presents the binarized image.Peaks are shown in Fig.4.c and Fig.4.d .

In the same manner, Fig.5.a presents a synthetic image containing two hyperbolas of equation $y=x^{3}$.The first is shifted by the vector $(10,0)$ and the second is shifted by the vector $(-10,10)$. The radon space $R_{1,0^{\circ}}$ shows two peaks related to the two hyperbolas position shift. 

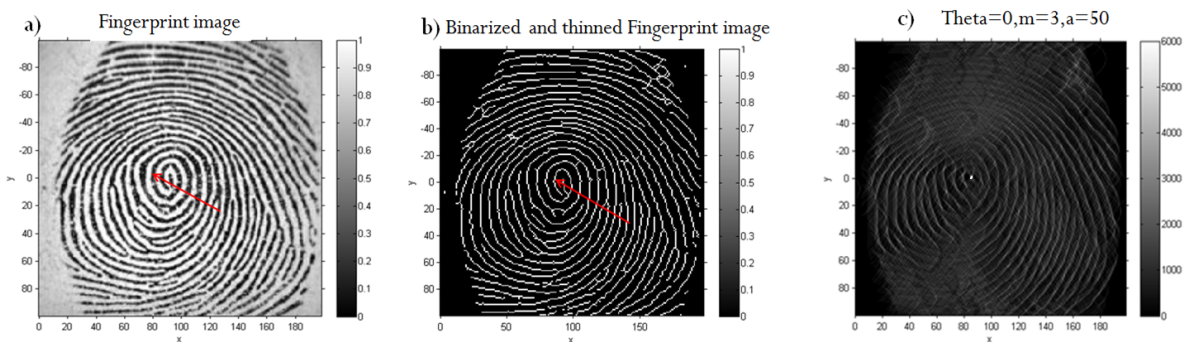

Fig. 3. a) A grey level fingerprint image where the vertex of the parabola we object to locate is pointed by a red arrow. (b) The binarized and thinned image. (c) The Radon space resulted from the application of the PDRT on the binarized and thinned image showing a peak at the vertex position pointed by the red arraw.
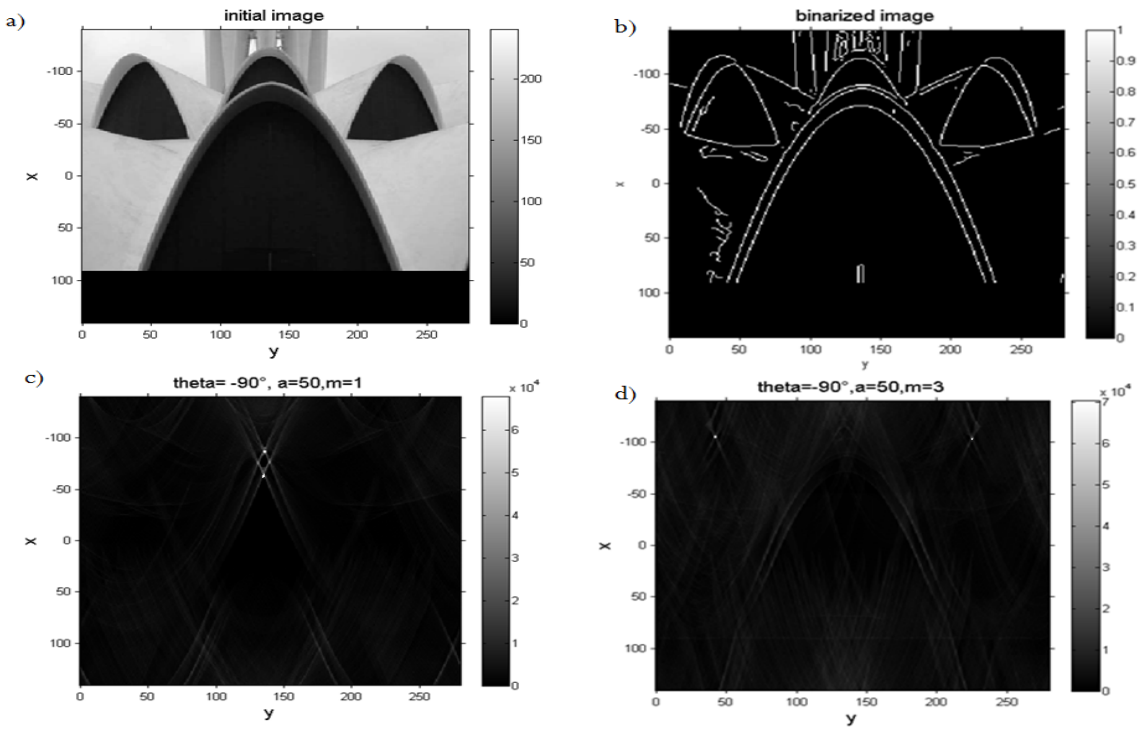

Fig. 4. a) Arches image. b) Binarized image. c) The Radon space applied on the binarized image, $\theta=-90^{\circ}, a=50, m=1$ showing two peaks at $(-90,140)$ and $(-60,140)$ d)The Radon space applied on the binarized image, $\theta=-90^{\circ}, a=50, m=3$ showing two symmetric peaks at $(-110,50)$ and $(-110,240)$.

We have also tested the inversion algorithm on several $p \times p$ sized images. The inversion was exact for each test. As an example, Fig.6 shows the result for an initial image which was projected with parabolic curves of equation $y=x^{2}$. 
a)

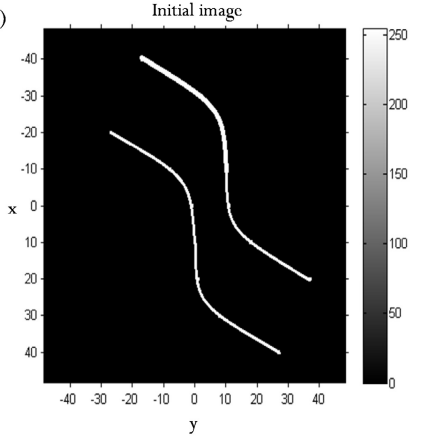

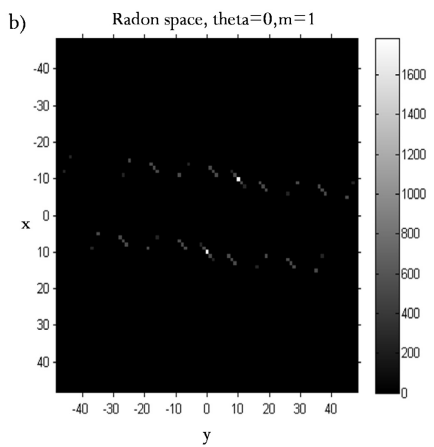

Fig. 5. a) : The initial image presenting two hyperbolas of equation $y=x^{3}$. The first at the left was translated from the origin $(0,0)$ by the vector $(\mathrm{v}, \mathrm{t})=(10,0)$ and the second was translated by the vector $(\mathrm{v}, \mathrm{t})=(-10,10)$. (b): The radon space for $\theta=0$ shows peaks at corresponding $(\mathrm{v}, \mathrm{t})$ positions.

a)

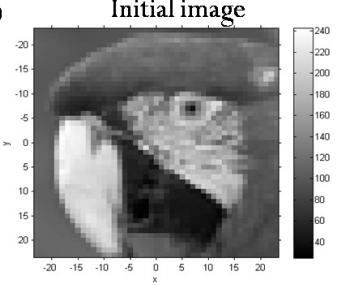

b) Radon space, theta $=0, m=1$

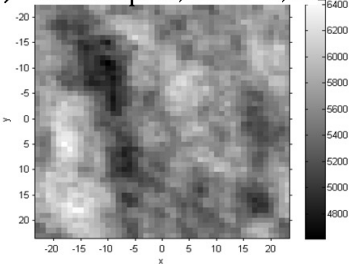

c) Reconstructed image

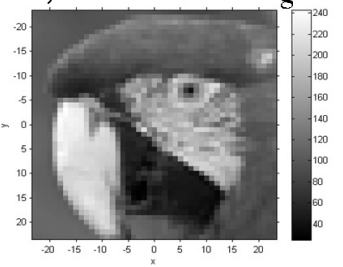

Fig. 6. Exact inversion illustration. (a):the initial image. (b) The polynomial Radon space performed by projecting parabolas where the projection angle $\theta=0$ and $m=1$. (c) The reconstructed image.

\section{Conclusion}

In this work, we have introduced a new approach that generalizes the classical Radon transform by allowing the projection of the image over polynomial curves following different directions. We showed through synthetic and real images the accuracy of the approach in detecting the orientation as well as the position of different polynomials in the image. An another advantage of the FPDRT approach is its exact invertibility. Therefore, the transform is particularly advantageous in applications which require both exact inversion and curves recognition. A possible application can be fingerprint or palmprint recognition where we need polynomial curves recognition for identifying persons and the exact inversion for encrypting images for security considerations. 


\section{References}

1. Deans, S.: The Radon Transform and Some of Its Applications. Krieger, Malabar (1993)

2. Courmontagne, P.: An improvement of ship wake detection based on the radon transform. Signal Processing 85 (2005)

3. Krishnaveni, M., Thakur, S.K., Subashini, P.: An Optimal Method For Wake Detection In SAR Images Using Radon Transformation Combined With Wavelet Filters. International Journal of Computer Science and Information Security 6, 66-69 (2009)

4. Zhang, Q., Couloigner, I.: Accurate Centerline Detection and Line Width Estimation of Thick Lines Using the Radon Transform. IEEE Transactions on Image Processing 16, 310-316 (2007)

5. Wang, L.Q., Hao, K., Radon Transform, Y.: Forstner Operator Applying in Buildings Contour Extraction. In: FSKD, pp. 415-419 (2009)

6. Magli, E., Olmo, G., Lo Presti, L.: Pattern recognition by means of the Radon transform and the continuous wavelet transform. Signal Processing 73, 277-289 (1999)

7. Rojbani, H., Elouedi, I., Hamouda, A.: R $\theta$-signature: A new signature based on Radon Transform and its application in buildings extraction. In: ISSPIT, pp. 490-495 (2011)

8. Deans, S.: Hough Transform from the Radon transform. IEEE Transactions On Pattern Analysis and Machine Intelligence, Pami-3, 185-188 (1981)

9. Tofts, P.: The Radon Ttransform: Theory and implementation. Ph.D.dissertation (1996)

10. Hendriks, C.L., van Ginkel, M., Verbeek, P.W., van Vliet, L.J.: The generalized Radon transform: Sampling, accuracy and memory considerations. Identification of Common Molecular Subsequences. Pattern Recognition 38, 2495-2505 (2005)

11. Beylkin, G.: Discrete Radon transform. IEEE Transactions on Acoustics, Speech and Signal Processing 35, 162-172 (1987)

12. Matus, F., Flusser, J.: Image representation via a Finite Radon transform. IEEE Transaction on Pattern Analysis and Machine Intelligence 15, 996-1006 (1993) 